\title{
BMJ Open Management of hyperglycaemia in persons with non-insulin-dependent type 2 diabetes mellitus who are started on systemic glucocorticoid therapy: a systematic review
}

\author{
Milos Tatalovic, ${ }^{1}$ Roger Lehmann, ${ }^{2}$ Marcus Cheetham, ${ }^{1,3}$ Albina Nowak, ${ }^{2}$ \\ Edouard Battegay, ${ }^{1,3}$ Silvana K Rampini ${ }^{1}$
}

To cite: Tatalovic M,

Lehmann R, Cheetham M, et al. Management of hyperglycaemia in persons with non-insulindependent type 2 diabetes mellitus who are started on systemic glucocorticoid therapy: a systematic review. BMJ Open 2019;9:e028914. doi:10.1136/ bmjopen-2019-028914

\section{- Prepublication history and} additional material are published online only. To view, please visit the journal online (http://dx.doi. org/10.1136/bmjopen-2019028914).

Received 2 January 2019 Revised 5 April 2019 Accepted 13 May 2019

Check for updates

(c) Author(s) (or their employer(s)) 2019. Re-use permitted under CC BY-NC. No commercial re-use. See rights and permissions. Published by BMJ.

${ }^{1}$ Department of Internal Medicine, University Hospital Zurich, Zurich, Switzerland ${ }^{2}$ Department of Endocrinology, Diabetes and Clinical Nutrition, University Hospital Zurich, Zurich, Switzerland

${ }^{3}$ Center of Competence Multimorbidity, University of Zurich, Zurich, Switzerland

Correspondence to Dr Silvana K Rampini; silvana.rampini@usz.ch

\section{ABSTRACT}

Objectives What is the most effective pharmacological intervention for glycaemic control in known type 2 diabetes mellitus (DM) without prior insulin treatment and newly started on systemic glucocorticoid therapy? Design We conducted a systematic literature review. Data sources We searched MEDLINE, Embase and Cochrane Library databases and Google for articles from 2002 to July 2018.

Eligibility criteria We combined search terms relating to DM (patients, $>16$ years of age), systemic glucocorticoids, glycaemic control, randomised controlled trials (RCTs) and observational studies.

Data extraction and synthesis We screened and evaluated articles, extracted data and assessed risk of bias and quality of evidence according to Grading of Recommendations Assessment, Development and Evaluation guidelines.

Results Eight of 2365 articles met full eligibility criteria. Basal-bolus insulin (BBI) strategy for patients under systemic glucocorticoid therapy was comparatively effective but provided insufficient glucose control, depending on time of day. BBI strategy with long-acting insulin and neutral protamin Hagedorn as basal insulin provided similar overall glycaemic control. Addition of various insulin strategies to standard BBI delivered mixed results. Intermediate-acting insulin (IMI) as additional insulin conferred no clear benefits, and glycaemic control with sliding scale insulin was inferior to BBI or IMI. No studies addressed whether anticipatory or compensatory insulin adjustments are better for glycaemic control. Conclusion The lack of suitably designed RCTs and observational studies, heterogeneity of interventions, targe glucose levels and glucose monitoring, poor control of DM subgroups and low to moderate quality of evidence render identification of optimal pharmacological interventions for glycaemic control and insulin management difficult. Even findings on the widely recommended BBI regimen as intensive insulin therapy for patients with DM on glucocorticoids are inconclusive. High-quality evidence from studies with well-defined DM phenotypes, settings and treatment approaches is needed to determine optimal pharmacological intervention for glycaemic control. PROSPERO registration number CRD42015024739.
Strengths and limitations of this study

- Systematic review with extensive literature search to provide comprehensive data on a very common but unresolved daily problem in managing type $2 \mathrm{di}-$ abetes mellitus (DM).

- Lack of comparability between studied populations and interventions and low to moderate quality of evidence does not permit full quantitative analysis and provision of formal recommendations on specific insulin regimens.

- Firm conclusions on optimal pharmacological interventions for glycaemic control awaits studies of sufficient power, quality and testing of well-defined DM phenotypes, settings and treatments.

\section{INTRODUCTION}

The worldwide prevalence of type 2 diabetes mellitus (DM) in adults has doubled since 1980 to $8.5 \%$ in $2014 .{ }^{1}$ While comparatively stable in recent years, the prevalence of hospitalised patients with DM is $25 \%-40 \% .^{23}$ Steroid treatment in patients with DM is common. ${ }^{45}$ However, steroids are the main cause of drug-induced hyperglycaemia ${ }^{6}$ due to their effect of increasing basal endogenous glucose production and lowering insulin sensitivity. ${ }^{7-9}$ Over half of patients receiving high-dose steroids develop hyperglycaemia. ${ }^{10}$ Significantly, steroids exacerbate hyperglycaemia in patients with pre-existing DM ${ }^{11} 12$ and enhance the likelihood of complications, length of stay and mortality in these patients. ${ }^{313-18}$

The importance of detecting and actively managing hyperglycaemia in patients with DM undergoing glucocorticoid therapy is acknowledged. ${ }^{13} 1920$ However, current management strategies are suboptimal, ${ }^{13} 21$ and the limited evidence available does not 
adequately inform the physician. ${ }^{6}$ This is all the more important as the type and doses of administered glucocorticoids and the potencies (and duration of action) of different systemic glucocorticoids vary widely. ${ }^{22-24}$ Shorter courses of steroids may lead to brief periods of hyperglycaemia that do not require further intervention, though hyperglycaemia and other side effects can occur at a wide range of doses. ${ }^{25}$ However, longer courses of steroids at higher doses can lead to symptomatic hyperglycaemia. ${ }^{2627}$ Optimal treatment strategies for glycaemic control are, therefore, vital.

The aim of this study was to conduct a systematic review of treatment strategies for glycaemic control in persons with type 2DM on diet or oral hypoglycaemic agents (OHA) and newly initiated glucocorticoid therapy. Specifically, we sought to identify the most effective pharmacological intervention for glycaemic control. We evaluated also whether the simultaneous start of insulin with glucocorticoids (anticipatory treatment adjustment) or delayed start of insulin, when blood glucose level (BGL) exceeds normal upper limits (compensatory treatment adjustment), is more effective.

\section{METHODS}

\section{Protocol and registration}

The review methods and eligibility criteria were specified in advance, documented in a study protocol, registered online with the International Prospective Register of Systematic Reviews, 31 May 2016, and recorded with a Preferred Reporting Items for Systematic Reviews and Meta-Analyses statement. ${ }^{28}$ We updated the protocol once on 21 October 2016 to broaden inclusion criteria.

\section{Eligibility criteria}

For eligibility, we followed the Patients, Interventions, Comparisons, Outcomes and Settings (PICOS) criteria. ${ }^{28}$ Patients: We included articles on non-critically ill (non-intensive care unit) in-patients or outpatients ( $>16$ years of age) who suffered from type $2 \mathrm{DM}$ treated with diet or OHA (ie, biguanide, gliflozins, gliptins, sulfonylureas, glinides, incretins or glitazones) and were started on a once or multiple daily oral or intravenous glucocorticoid therapy (ie, hydrocortisone, prednisone, prednisolone, methylprednisolone, dexamethasone, betamethasone or fludrocortisone) irrespective of the indication. Interventions: The articles and studies had to address specific treatment interventions for glycaemic control, including, for example, stopping routine DM medication and starting insulin treatment. Comparisons: We included all types of comparisons of the study population with those (1) without DM, (2) without glucocorticoid treatment, (3) with adjusted OHA, or (4) with differing insulin treatments. Outcomes: We accepted outcomes reflecting glycaemic control, that is, time outside target glucose range, mean BGL, hypoglycaemic or hyperglycaemic episodes, and daily insulin dose. Settings: We included randomised controlled trials (RCTs) and observational studies, that is, cohort studies, case-control studies or cross-sectional studies, without imposing any restriction on language, country of origin or publication type. We excluded letters to the editor and conference abstracts. We consulted also guidelines, reviews and expert opinions. We considered only papers published after 2002 because of the subsequent introduction of long-acting insulin; long-acting insulins are, nowadays, an integral part of treatment in insulin-dependent DM.

\section{Search strategy}

We identified articles based on search terms related to $\mathrm{DM}$ and glucocorticoids in the following databases: MEDLINE and PreMEDLINE using OVID, Embase and Cochrane Library electronic databases (online supplementary table 1). The combined use of the databases (PubMed, MEDLINE, Embase and Cochrane) allows coverage of up to $97 \%$ of available publications. ${ }^{29}$ To enhance coverage further, we conducted also a Google search to retrieve grey literature with exclusive focus on pdf files. The search was conducted on 8 July 2016 and updated on 2 July 2018.

\section{Study selection}

MT and SKR independently screened a sample of 100 papers by studying the titles and abstracts according to the selection criteria 'adult persons with pre-existing DM who received a glucocorticoid therapy'. If no abstract was available but the title appeared relevant, MT and SKR reviewed the full text. One abstract was translated from Japanese.

MT and SKR then evaluated the first 100 papers in consensus to establish the basis for consistent screening of all further papers. MT performed the screening of all papers and SKR independently double screened a random sample of $10 \%$ of all articles. All articles were assigned to one of the three eligibility groups: 'yes', 'no' and 'maybe'. The 'maybe' group was discussed by MT and SKR for eligibility after full-text review in a consensus conference. Initial review of eligible articles revealed the necessity for modification of the inclusion criterion ' $\geq 20 \mathrm{mg} /$ day prednisolone equivalent for $\geq 5$ days' to 'intermediate-dose or high-dose glucocorticoid therapy' because a large number of articles did not specify exact dosages of glucocorticoids.

MT and SRK independently performed a full-text review of all eligible papers for inclusion, considering the PICOS criteria. Disagreements between reviewers were resolved by consensus. Finally, the reference lists of all included articles were screened for additional eligible papers, guidelines and review articles.

\section{Data extraction and quality assessment}

We extracted the following data from the included articles: study population, participants, and age. Then, we assessed indication, dosage and duration of glucocorticoid therapy, target glucose, insulin strategy, the management of OHA interruption, continuation or adjustment 
of dosages and outcome measures, such as time in target glucose range, mean BGL, hypoglycaemic and hyperglycaemic episodes and insulin requirement. Differing assessments were discussed and resolved between MT and SKR.

We used the Cochrane risk of bias tool ${ }^{30}$ to evaluate the risk of bias in RCTs and applied the key criteria of the Grading of Recommendations Assessment, Development and Evaluation (GRADE) guidelines for observational studies to assess the methodological quality of nonrandomised studies. ${ }^{31}$ The overall quality of evidence was assessed using the GRADE criteria. ${ }^{32}$

\section{Data synthesis}

We performed a descriptive analysis of RCTs and observational studies. This was because the lack of concordance in the study designs in the included articles precluded the performance of meta-analyses. Included articles were evaluated and compared in detail and the findings were summarised.

\section{Patient or public involvement}

Neither the patients nor the public were directly involved in the development of the research question, selection of the outcome measures, design and implementation of the study, or interpretation of the results.

\section{RESULTS}

\section{Study inclusion}

Our initial search generated 3521 articles. A total of 2365 articles remained after eliminating duplicate entries. Of these, 37 qualified for full-text review. Eight articles met full eligibility criteria, namely, four RCTs ${ }^{33-36}$ with openlabel and parallel group designs, one RCT with openlabel and cross-over design ${ }^{37}$ and three observational studies $^{38-40}$ with a retrospective cohort design (figure 1 and table 1).

The eight articles reported studies that included a total of 481 persons, $343 / 481$ persons with DM and $138 / 481$ persons with glucocorticoid-induced hyperglycaemia. One study included persons with both type 1 and type $2 \mathrm{DM}$ but did not take this distinction into consideration for outcomes. ${ }^{33}$ At least $85 / 481$ persons had prior treatment with insulin; three studies did not provide this information. ${ }^{33} 3439$ Seven studies included inpatients only, ${ }^{33-3638-40}$ and one study included both inpatients and outpatients. ${ }^{37}$ Capillary blood glucose was measured four times a day by continuous glucose monitoring or by using all available capillary and serum blood glucose readings (table 2). The upper limit was a BGL of $10 \mathrm{mmol} / \mathrm{L}$ in all studies. The lower BGL limit was $3.9-4.5 \mathrm{mmol} / \mathrm{L}$ in all but two studies in which it was $5.6 \mathrm{mmol} / \mathrm{L}^{33} 40$ (table 2). Insulin dose adjustments were applied if BGL was outside the target glucose range, according to specific study protocols.

In six studies, authors treated control groups with a basal-bolus insulin (BBI) regimen using insulin glargine as basal insulin, ${ }^{33-363839}$ in one study with a BBI regimen using twice-daily insulin detemir, ${ }^{40}$ and one study using sliding-scale insulin (SSI) in addition to established DM medication. ${ }^{37}$ Strikingly, treatment interventions in the experimental groups diverged substantially. One study compared glycaemic control of BBI regimen in patients with type $2 \mathrm{DM}$ without prednisolone with those with prednisolone treatment. ${ }^{38}$ Another study compared glycaemic control of BBI regimen with SSI regimen. ${ }^{40}$ One study compared addition of SSI to routine DM medication with the addition of intermediate-acting insulin (IMI). ${ }^{37}$ Three studies compared BBI regimens with long-acting insulins to BBI regimens with intermediate-acting neutral protamin Hagedorn (NPH) insulin, ${ }^{35} 3639$ but in one of these studies, NPH was given in three equal prandial doses. ${ }^{36}$ One study compared BBI regimen with longacting insulin to the same regimen with the addition of NPH insulin. ${ }^{34}$ Finally, the most recent study added the insulin type that matched the glycaemic profile of the administered glucocorticoid. ${ }^{33}$ This divergence in study designs of RCTs precluded a clean and coherent quantitative meta-analysis.

BBI strategy in persons under systemic glucocorticoid therapy Two observational studies ${ }^{38} 40$ report BBI as superior in glucocorticoid-treated patients with type 2DM. ${ }^{41}{ }^{42}$ Gosmanov et $a t^{40}$ found more hyperglycaemic events in patients with type $2 \mathrm{DM}$ under dexamethasone for 3 days (for a hematological malignancy) when treated with SSI therapy compared with a BBI therapy (table 2). In the SSI group, the mean daily BGL was significantly higher $(\mathrm{p}<0.001)$, and the average insulin requirement was significantly lower $(\mathrm{p}<0.001)$. No hypoglycaemic events occurred in either groups, but $3 / 28$ (11\%) persons treated with SSI were referred to an intensive care unit because of hyperglycaemic events.

Burt $e t a l^{38}$ studied the effectiveness of a BBI regimen in hospitalised patients with type $2 \mathrm{DM}$ treated with prednisolone in the morning for an acute medical condition compared with those without glucocorticoid treatment. Half of the calculated daily dose was given as long-acting insulin glargine at 21:00 and half as bolus evenly split into three meal dosages of rapid-acting insulin with additional correctional insulin when necessary. The mean daily BGL was significantly higher in the prednisolone group $(\mathrm{p}<0.001)$ (table 2$)$. More specifically, BGL was significantly higher at 17:00 and 21:00, but not significantly higher at 07:00 and 12:00 In addition, the daily insulin dose was significantly higher in the prednisolone-treated group than in the control group, especially at 12:00 and 17:00. Thus, BBI treatment provided insufficient glucose control, most notably in the afternoon and evening.

Comparison of BBI regimen with long-acting insulin to NPH as basal insulin

Two RCTs ${ }^{35} 36$ and one observational study ${ }^{39}$ compared $\mathrm{NPH}$ insulin with the long-acting insulin glargine in a BBI regimen for their efficacy in controlling BGL in 

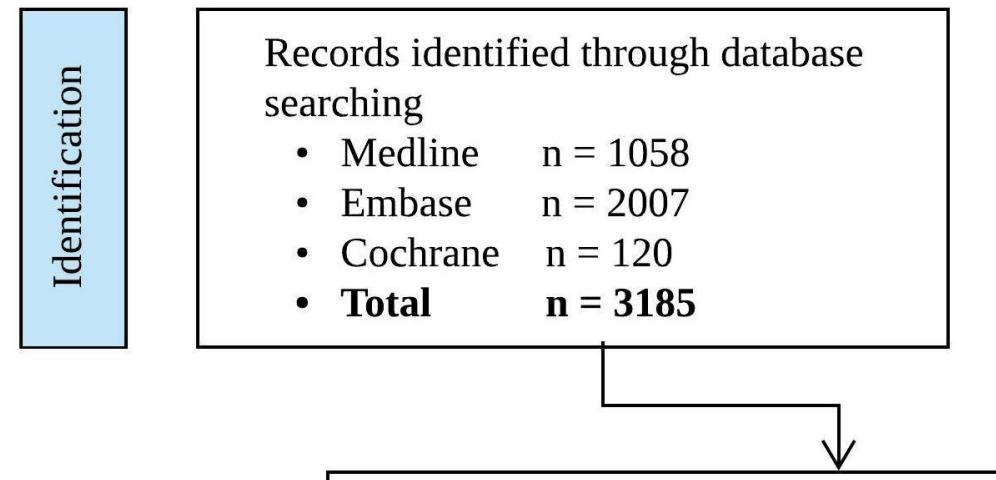

Additional records identified from grey literature

- Google $\mathbf{n}=\mathbf{3 3 6}$

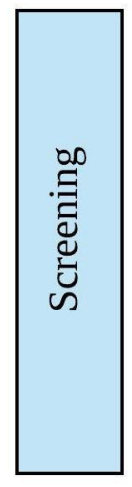

Records after duplicates removed

Medline n=438; Embase n=1820; Cochrane n=101; Google n=6
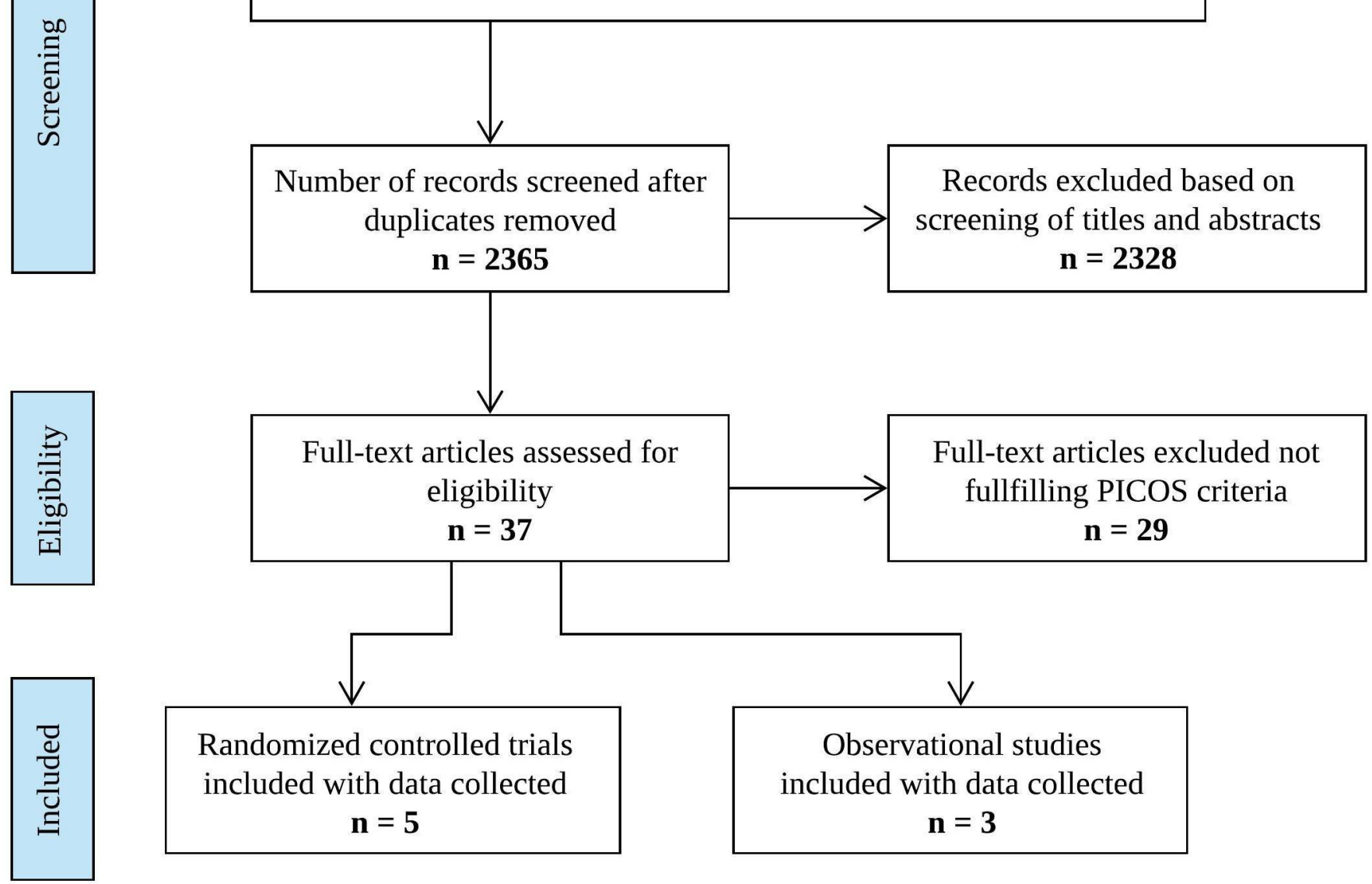

Figure 1 Flow diagram of study selection. PICOS, patients, interventions, comparisons, outcomes and settings.

hospitalised persons treated with medium-dose to highdose glucocorticoids. ${ }^{36}{ }^{39}$ The studies differed substantially in their design (table 1 ). Radhakutty et $a l^{35}$ included persons with or without type $2 \mathrm{DM}$ who were treated with a single dose of glucocorticoids for respiratory disease or gout. Glargine was administered in the control group and $\mathrm{NPH}$ in the experimental group at 07:00. Ruiz de Adana et $a l^{36}$ studied patients with type $2 \mathrm{DM}$ receiving multiple daily doses of glucocorticoids for respiratory disease. The glargine group received its basal insulin as one dose at 09:00, and the NPH group received it before breakfast, lunch and dinner in three equal doses. Dhital $e t a l^{99}$ retrospectively studied adults treated with prednisone who were on a BBI regimen with either insulin glargine or NPH. Notably, the target glucose range, the time of application and the number of doses of basal insulins were not indicated here, and persons with hyperglycaemia without underlying type 2DM were also included.

All three studies show a similar overall glycaemic control for NPH or glargine as basal insulin. ${ }^{35} 3639$ More specifically, the mean daily BGL and the number of mild hypoglycaemic episodes per day were similar (table 2). Notably, severe hypoglycaemia (BGL $<2.22 \mathrm{mmol} / \mathrm{L}$ ) occurred in two persons in the NPH group in the study by Ruiz de Adana $e t a l .{ }^{36}$ Only Dhital $e t a l^{99}$ found a significantly lower daily insulin requirement in the NPH group. 


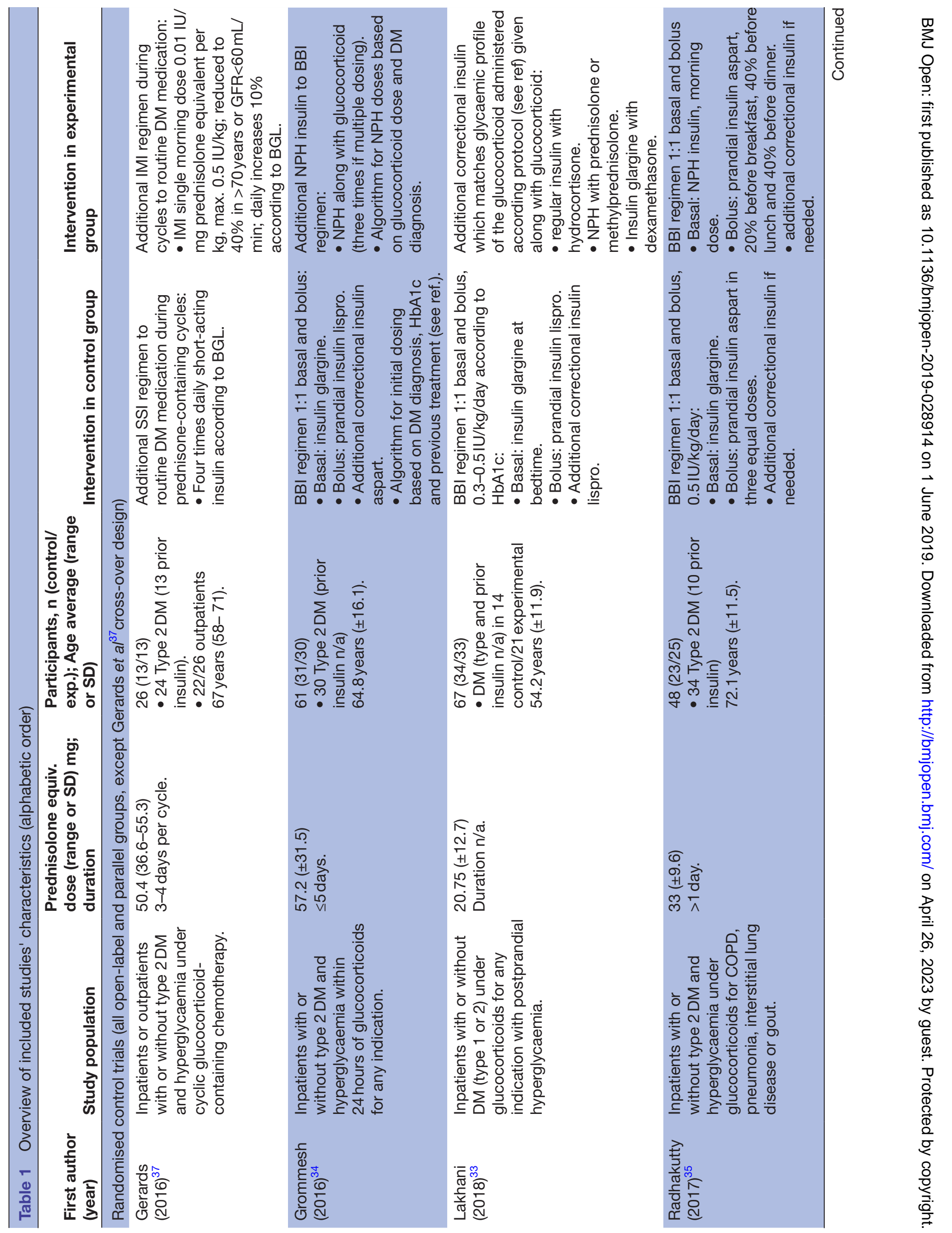




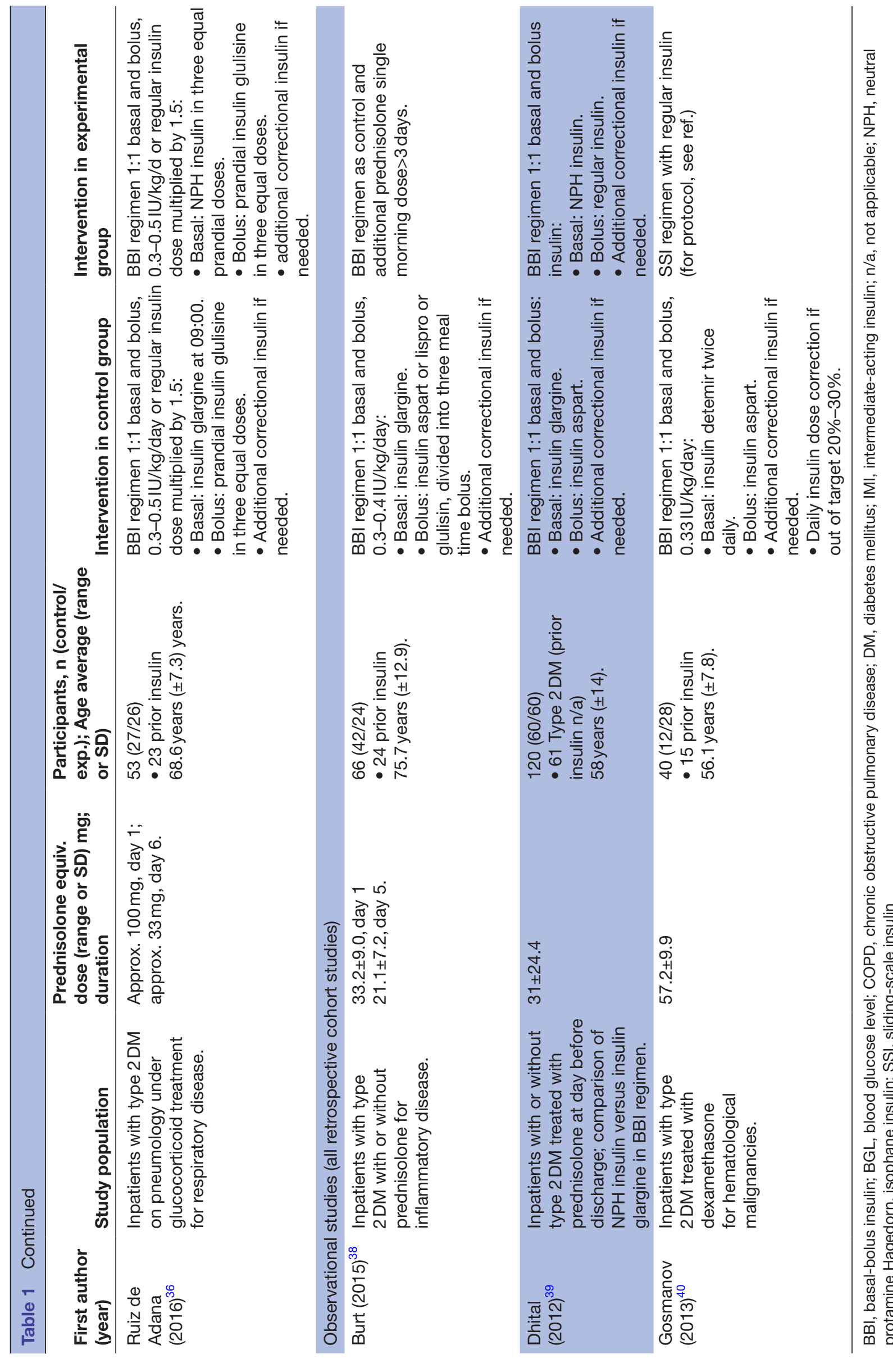




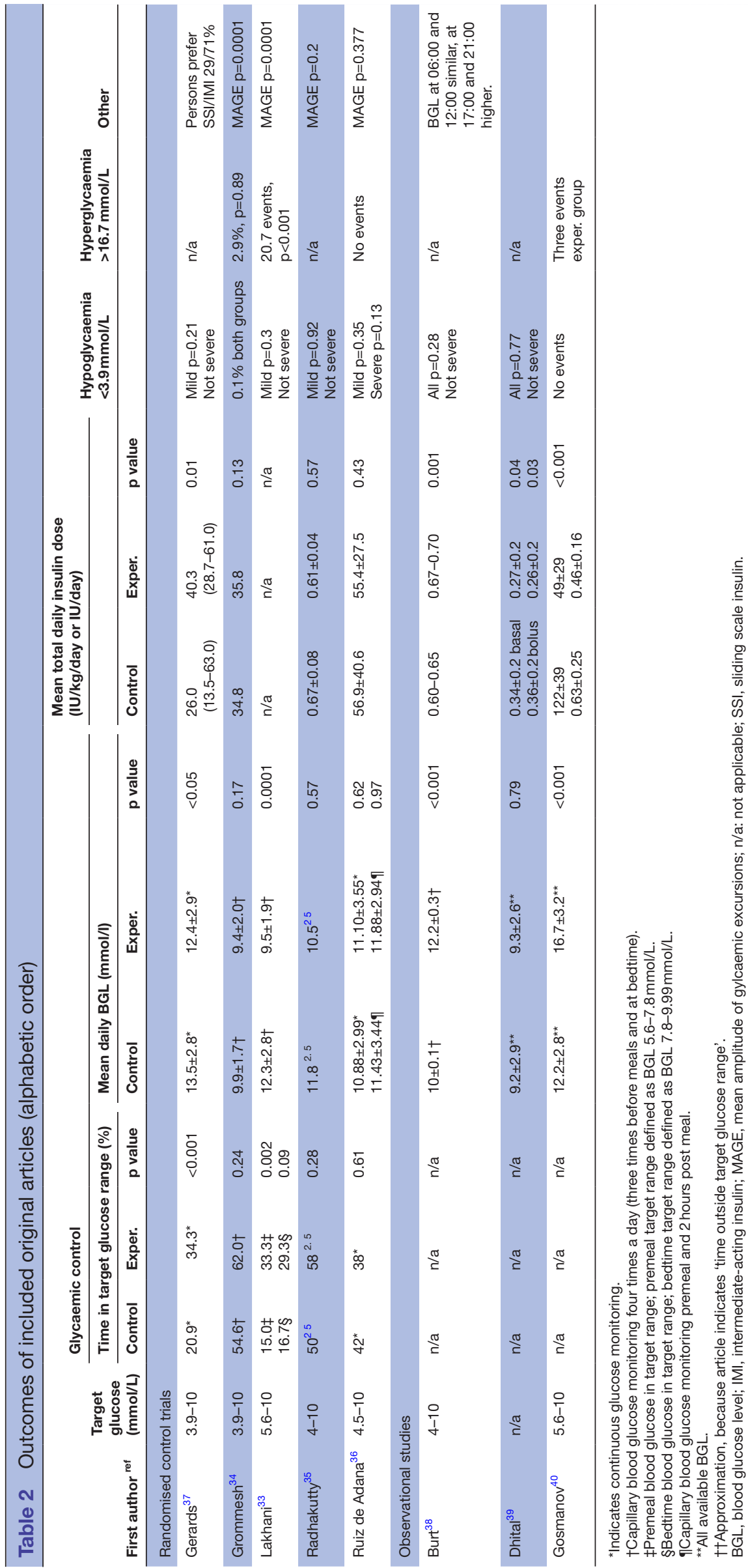

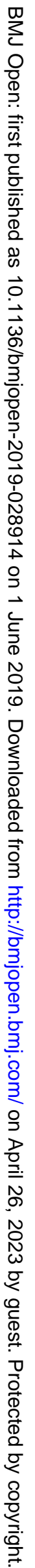




\section{Addition of insulin to established DM medication}

Gerards $e t a l^{37}$ compared the addition of SSI insulin compared with IMI to established DM medication for glycaemic control. The types of insulin were not further defined. Half of the persons had prior insulin treatment. The addition of IMI resulted in significantly longer time in target glucose range $(\mathrm{p}<0.001)$ and lower mean daily BGL $(\mathrm{p}<0.05)$. This was achieved with an increased insulin requirement in the IMI group. Remarkably, the mean daily BGL of both groups (SSI 13.5 \pm 2.8 , IMI 12.4 \pm 2.9 ) was higher than those of all other studies (table 2).

Two RCTs added insulin to an existing BBI regimen in persons with or without type $2 \mathrm{DM}^{33} 34$ (table 1 ). Grommesh $e$ t $a l^{34}$ studied the addition of NPH insulin together with a glucocorticoid to a BBI regimen. The algorithm for NPH dosing was based on glucocorticoid type, dose and pre-existing DM diagnosis. The study showed that there was no advantage in this for glycaemic control, mean total daily insulin dose, or hypoglycaemia and hyperglycaemia (table 2). Similarly, an RCT by Lakhani et a ${ }^{33}$ studied the addition of a so-called 'correctional insulin' together with the glucocorticoid to a BBI regimen. The type of correctional insulin matched the glycaemic profile of the type of administered glucocorticoid, for example, $\mathrm{NPH}$ insulin for prednisolone or insulin glargine for dexamethasone treatment. ${ }^{33}$ Correctional insulin significantly improved 'time in target premeal glucose range' (defined as $5.6-10 \mathrm{mmol} / \mathrm{L}(\mathrm{p}=0.002)$ and mean daily BGL $(p=0.0001)$ but not time in 'bedtime target glucose range' $(p=0.09)$. The hyperglycaemic events were reduced $(p<0.001)$. No data were provided on subgroups without $\mathrm{DM}$ or with type $1 \mathrm{DM}$ and on daily insulin doses.

\section{Anticipatory or compensatory approach to glycaemic control}

We aimed to determine whether anticipatory or compensatory adjustments are better for glycaemic control. No screened or included study addressed this issue. While screening articles, we found some recommendations about this in guidelines ${ }^{41} 43-45$ and reviews, ${ }^{46-50}$ and we address this issue in the discussion section.

\section{Risk of bias and grading of evidence}

Risk of bias was assessed in five RCTs for seven domains and four outcomes (mean BGL, time in target glucose, daily insulin dose and hypoglycaemia) (online supplementary table 2a). All RCTs were unblinded for participants and personnel. Although placebo effects are very unlikely, unblinding may have affected the attention of staff. This might be the most relevant risk for bias in these studies. The lack of random sequence generation and allocation concealment might be another common bias. The three observational studies were classified as having low, ${ }^{38}$ middle $^{39}$ and high ${ }^{40}$ ranges of risk of bias (online supplementary table $2 \mathrm{~b}$ ). The most common risk of bias was the failure to control confounding. Notably, an overall risk of bias of an outcome for all five RCTs is not so informative because the treatment interventions were not comparable.
Applying the GRADE criteria on each individual study, we had to decrease the level of evidence for the primary outcomes 'mean BGL' and 'time in target glucose range.' This was mainly because of risk of bias and publication bias and because of inconsistency and imprecision in the five RCTs and one observational study (for the overall rating of quality of evidence in RCTs and observational studies, see online supplementary table 3). Hence, we classified the overall quality of evidence for the individual interventions as moderate, ${ }^{33} 3637$ low 3538 or very low. ${ }^{34} 40$

\section{DISCUSSION}

Glucocorticoid treatment inevitably leads to hyperglycaemia in persons with type 2DM. We systematically reviewed the available evidence on pharmacological interventions for effective glycaemic control. We found, first, that there is some uncertainty as to the optimal management of glucocorticoid-induced hyperglycaemia in DM. The lack of studies reporting high-quality evidence makes it difficult to provide formal and final recommendations. This review shows that the available evidence is of low to moderate quality. Second, the reviewed studies speak in favour of the use of BBI without a specific preference for long-acting or IMI as basal insulin, but these studies do indicate that SSI should be abandoned. Third, two studies suggested that pharmacodynamic profiles of insulins should be reconciled with corresponding profiles of glucocorticoids. However, there is insufficient evidence to recommend this. Finally, the reviewed studies do not clarify whether one should initiate anticipatory or compensatory insulin treatment.

$\mathrm{BBI}$ is widely accepted and recommended as intensive insulin therapy in DM. ${ }^{42} 5152$ However, the question remains whether BBI performs best in type 2DM under glucocorticoid treatment. Five open-label RCTs and three observational studies included in this systematic review address this issue. Gosmanov et $a l^{40}$ shows that BBI is better than SSI in terms of glycaemic control. This is in line with data from various clinical settings that support improved hyperglycaemic control using BBI compared with SSI. ${ }^{53}$ Gerards $e t a l^{37}$ corroborates that SSI delivers poorer control compared with IMI when used as an addition to the routine DM regimen. Although very popular among non-endocrinologists, these findings suggest that SSI treatment should not be prescribed in this setting anymore. On the other hand, Burt $e t a \hat{l}^{8}$ did find that glycaemic control was insufficient at 17:00 and 21:00 when using BBI with long-acting insulin in patients with type $2 \mathrm{DM}$ treated with prednisolone compared with those without prednisolone treatment. These findings are in line with previous reports of afternoon and evening hyperglycaemia under glucocorticoids in persons without DM. ${ }^{242755}$ Thus, BBI with long-acting insulin does not offer a final solution.

The intermediate-acting NPH insulin provides good control of afternoon peaks of blood glucose caused by glucocorticoids. This approach might have an advantage 
over long-acting insulin because its effects show a similar timeline to that of glucocorticoid-induced afternoon peaks of hyperglycaemia. ${ }^{56}$ Three of the reviewed articles $^{35} 3639$ compared NPH insulin with insulin glargine as basal insulin in a BBI treatment in randomised controlled $^{35} 36$ and retrospective ${ }^{39}$ studies, finding no significant differences in glycaemic control. However, NPH insulin caused more hypoglycaemic events when $\mathrm{NPH}$ and bolus insulin were administered in equal preprandial doses for the purpose of controlling hyperglycaemia in persons receiving multiple daily doses of glucocorticoids. ${ }^{36}$ Such a protocol may not be flexible enough in that it does not give sufficient consideration to the night-time fasting period and the associated risk of nocturnal hypoglycaemia. Insulin requirement, however, was higher in BBI with long-acting insulin compared with NPH as basal insulin in two of the studies, ${ }^{36}{ }^{39}$ but it was similar in the other. ${ }^{35}$ The addition of NPH together with the glucocorticoid in the BBI treatment also failed to improve glycaemic control. ${ }^{34}$ The most recent study by Lakhani $e t a l^{33}$ suggests a unique approach to better match the pharmacodynamic properties of insulins and glucocorticoids. This resulted in significantly lower mean daily BGL and premeal time in target glucose range. While the approach of Lakhani $e t a l^{33}$ appears to be promising, it does need to be corroborated in a larger study.

We found no primary data comparing anticipatory with compensatory treatment adjustments for glycaemic control when starting glucocorticoids. This lack of data is a source of some divergence in expert opinions in guidelines. The American Endocrine Society Clinical Practice Guidelines ${ }^{41}$ recommends an anticipatory approach with discontinuation of OHA at the time of hospital admission and initiation of insulin with persistent hyperglycaemia. In exceptional cases, selected persons who are stable, eating regularly and have no contraindication 'may be candidates for continuation of previously prescribed OHA'. The Canadian Diabetes Association guideline ${ }^{45}$ recommends that 'glycemic monitoring for 48 hours after initiation of steroids may be considered'. In contrast, the Joint British Diabetes Societies for inpatient care guideline, ${ }^{19}$ and the Imperial College Clinical Guidance ${ }^{58}$ recommend the up-titration of OHA first. They recommend adding ${ }^{19}$ or switching ${ }^{58}$ to insulin if BGL remains above $10 \mathrm{mmol} / \mathrm{L}$. Reports of experience or evidence to suggest the use of DDP-4 inhibitors, GLP-1 receptor agonists or SGLT-2 inhibitors are lacking.

The strength of our systematic review is that it makes an important contribution to DM management. It does this by highlighting the unresolved challenge of good glycaemic control in patients with DM who are on systemic glucocorticoid therapy and by reporting an extensive literature search on hyperglycaemic control in these patients. However, we cannot draw conclusions from our systematic analysis on the most effective management approach. This is largely due to the low to moderate quality of available evidence and the lack of comparability between the reviewed studies. In fact, this review draws attention to the heterogeneity of the experimental designs and the lack of well-powered, high-quality studies. Given that the populations, interventions, target glucose levels and glucose monitoring differed from study to study, the main limitation is that we can only provide a descriptive review of the studies but not formal recommendations. Well-designed studies with more homogeneous patient populations are needed in order to answer the questions raised in this review. The present review focused on the population of persons with pre-existing type 2DM without prior insulin treatment. However, we included articles with mixed populations, namely, persons with type 2 DM with or without prior insulin treatment and type $1 \mathrm{DM}$, because there is an insufficient number of articles with the specific subgroup of interest. We acknowledge that this is not standard practice in systematic reviews.

\section{CONCLUSION}

Glucocorticoid therapy exacerbates hyperglycaemia in patients with pre-existing DM. Current management strategies give insufficient guidance for glycaemic control in persons started on glucocorticoids. The lack of relevant RCTs and observational studies, heterogeneity of populations, interventions, target glucose levels and glucose monitoring in available studies, and low to moderate quality of available evidence make it difficult to identify pharmacological interventions for effective glycaemic control. Even for the widely recommended use of a BBI regimen as intensive insulin therapy in DM, the data on this regimen in patients with DM on glucocorticoids are inconclusive. Indeed, the findings of our systematic review clearly speak in favour of the call to action on research in inpatient DM management of The PRIDE group $^{59}$ and in outpatient care. A concerted effort of diabetes societies would be needed to develop powerful study designs that take into account different DM phenotypes, settings and treatment approaches.

Contributors MT drafted the study; contributed to the development of the selection criteria and data extraction criteria; developed the search strategy; elaborated the study selection, data extraction and data synthesis; wrote the manuscript; provided feedback and approved the final manuscript. RL provided expertise on diabetes mellitus, read the manuscript, provided feedback and approved the final manuscript. MC contributed to the writing of the manuscript. AN read, provided feedback and approved the final manuscript. EB drafted the study, contributed to the development of the selection criteria and data extraction criteria, read the manuscript, provided feedback and approved the final manuscript. SKR is the guarantor; drafted the study; contributed to the development of the selection criteria and data extraction criteria; did the study selection, data extraction and data synthesis; wrote the manuscript; provided feedback and approved the final manuscript.

Funding The authors have not declared a specific grant for this research from any funding agency in the public, commercial or not-for-profit sectors.

Competing interests None declared.

Patient consent for publication Not required.

Provenance and peer review Not commissioned; externally peer reviewed.

Data sharing statement All data relevant to the study are included in the article or uploaded as supplementary information.

Open access This is an open access article distributed in accordance with the Creative Commons Attribution Non Commercial (CC BY-NC 4.0) license, which 
permits others to distribute, remix, adapt, build upon this work non-commercially, and license their derivative works on different terms, provided the original work is properly cited, appropriate credit is given, any changes made indicated, and the use is non-commercial. See: http://creativecommons.org/licenses/by-nc/4.0/.

\section{REFERENCES}

1. WHO. Global Health Report on Diabetes. 2016:1-87.

2. Digital NHS. National Diabetes Inpatient Audit (NaDIA) - 2016. 2017 http://www.content.digital.nhs.uk/catalogue/PUB23539.

3. Umpierrez GE, Isaacs SD, Bazargan N, et al. Hyperglycemia: an independent marker of in-hospital mortality in patients with undiagnosed diabetes. J Clin Endocrinol Metab 2002;87:978-82.

4. Hans P, Vanthuyne A, Dewandre PY, et al. Blood glucose concentration profile after $10 \mathrm{mg}$ dexamethasone in non-diabetic and type 2 diabetic patients undergoing abdominal surgery. $\mathrm{Br} \mathrm{J}$ Anaesth 2006;97:164-70.

5. Fardet L, Petersen I, Nazareth I. Prevalence of long-term oral glucocorticoid prescriptions in the UK over the past 20 years. Rheumatology 2011;50:1982-90.

6. Tamez-Pérez HE, Quintanilla-Flores DL, Rodríguez-Gutiérrez R, et al. Steroid hyperglycemia: Prevalence, early detection and therapeutic recommendations: A narrative review. World J Diabetes 2015:6:1073-81.

7. van Raalte DH, Nofrate V, Bunck MC, et al. Acute and 2-week exposure to prednisolone impair different aspects of beta-cell function in healthy men. Eur J Endocrinol 2010;162:729-35.

8. van Raalte DH, Brands M, van der Zijl NJ, et al. Low-dose glucocorticoid treatment affects multiple aspects of intermediary metabolism in healthy humans: a randomised controlled trial. Diabetologia 2011;54:2103-12.

9. Geer EB, Islam J, Buettner C. Mechanisms of glucocorticoidinduced insulin resistance: focus on adipose tissue function and lipid metabolism. Endocrinol Metab Clin North Am 2014;43:75-102.

10. Fong AC, Cheung NW. The high incidence of steroid-induced hyperglycaemia in hospital. Diabetes Res Clin Pract 2013;99:277-80.

11. van Raalte DH, Ouwens DM, Diamant M. Novel insights into glucocorticoid-mediated diabetogenic effects: towards expansion of therapeutic options? Eur J Clin Invest 2009;39:81-93.

12. Perez A, Jansen-Chaparro S, Saigi I, et al. Glucocorticoid-induced hyperglycemia. J Diabetes 2014;6:9-20.

13. Wallymahmed ME, Dawes S, Clarke G, et al. Hospital in-patients with diabetes: increasing prevalence and management problems. Diabet Med 2005;22:107-9.

14. Khalid JM, Raluy-Callado M, Curtis $\mathrm{BH}$, et al. Rates and risk of hospitalisation among patients with type 2 diabetes: retrospective cohort study using the UK General Practice Research Database linked to English Hospital Episode Statistics. Int J Clin Pract 2014;68:40-8.

15. Pomposelli JJ, Baxter JK, Babineau TJ, et al. Early postoperative glucose control predicts nosocomial infection rate in diabetic patients. JPEN J Parenter Enteral Nutr 1998;22:77-81.

16. Menzin J, Korn JR, Cohen J, et al. Relationship between glycemic control and diabetes-related hospital costs in patients with type 1 or type 2 diabetes mellitus. J Manag Care Pharm 2010;16(4):264-75.

17. Bloom RD, Crutchlow MF. Transplant-associated hyperglycemia. Transplant Rev 2008;22:39-51.

18. Inzucchi SE. Management of Hyperglycemia in the Hospital Setting. N Engl J Med Overseas Ed 2006;355:1903-11.

19. Roberts A, James J, Dhatariya K; Joint British Diabetes Societies (JBDS) for Inpatient Care. Management of hyperglycaemia and steroid (glucocorticoid) therapy: a guideline from the Joint British Diabetes Societies (JBDS) for Inpatient Care group. Diabet Med 2018;35:1011-7.

20. Jannot-Lamotte MF, Raccah D. [Management of diabetes during corticosteroid therapy]. Presse Med 2000;29:263-6.

21. Hirsch IB. Sliding scale insulin--time to stop sliding. JAMA 2009;301:213-4.

22. Ericson-Neilsen W, Kaye AD. Steroids: pharmacology, complications, and practice delivery issues. Ochsner J 2014;14:203-7.

23. National Institute for Health and Clinical Excellence (NICE) Clinical Knowledge Summaries: Corticosteroids - Oral. NICE $2012 \mathrm{http} / / /$ www.cks.nhs.uk/corticosteroids_oral.

24. Yuen KC, McDaniel PA, Riddle MC. Twenty-four-hour profiles of plasma glucose, insulin, C-peptide and free fatty acid in subjects with varying degrees of glucose tolerance following short-term, medium-dose prednisone $(20 \mathrm{mg} /$ day) treatment: evidence for differing effects on insulin secretion and action. Clin Endocrinol 2012;77:224-32.
25. Kendall EC. Nobel lecture: The development of cortisone as a therapeutic agent. Nobelprizeorg 1950 http://www.content.digital. nhs.uk/catalogue/PUB23539.

26. Donihi AC, Raval D, Saul M, et al. Prevalence and predictors of corticosteroid-related hyperglycemia in hospitalized patients. Endocr Pract 2006;12:358-62.

27. Burt MG, Roberts GW, Aguilar-Loza NR, et al. Continuous monitoring of circadian glycemic patterns in patients receiving prednisolone for COPD. J Clin Endocrinol Metab 2011;96:1789-96.

28. Shamseer L, Moher D, Clarke M, et al. Preferred reporting items for systematic review and meta-analysis protocols (PRISMA-P) 2015: elaboration and explanation. BMJ 2015;349:97647.

29. Harris JD, Quatman CE, Manring MM, et al. How to write a systematic review. Am J Sports Med 2014;42:2761-8.

30. Higgins JP, Altman D. Assessing the risk of bias in included studies. In: Higgins JPT, Green S, eds. Cochrane Handbook for Systematic Reviews of Interventions Version 510 [updated March 2011]: The Cochrane Collaboration2011-33-49.

31. Guyatt GH, Oxman AD, Vist G, et al. GRADE guidelines: 4. Rating the quality of evidence--study limitations (risk of bias). J Clin Epidemiol 2011;64:407-15.

32. Guyatt G, Oxman AD, Akl EA, et al. GRADE guidelines: 1 . Introduction-GRADE evidence profiles and summary of findings tables. J Clin Epidemiol 2011;64:383-94.

33. Lakhani OJ, Kumar S, Tripathi S, et al. Comparison of Two Protocols in the Management of Glucocorticoid-induced Hyperglycemia among Hospitalized Patients. Indian J Endocrinol Metab 2017;21:836-44.

34. Grommesh B, Lausch MJ, Vannelli AJ, et al. Hospital insulin protocol aims for glucose control in glucocorticoid-induced hyperglycemia. Endocr Pract 2016;22:180-9.

35. Radhakutty A, Stranks JL, Mangelsdorf BL, et al. Treatment of prednisolone-induced hyperglycaemia in hospitalized patients: Insights from a randomized, controlled study. Diabetes Obes Metab 2017;19:571-8

36. Ruiz de Adana MS, Colomo N, Maldonado-Araque C, et al. Randomized clinical trial of the efficacy and safety of insulin glargine vs. NPH insulin as basal insulin for the treatment of glucocorticoid induced hyperglycemia using continuous glucose monitoring in hospitalized patients with type 2 diabetes and respiratory disease. Diabetes Res Clin Pract 2015;110:158-65.

37. Gerards MC, de Maar JS, Steenbruggen TG, et al. Add-on treatment with intermediate-acting insulin versus sliding-scale insulin for patients with type 2 diabetes or insulin resistance during cyclic glucocorticoid-containing antineoplastic chemotherapy: a randomized crossover study. Diabetes Obes Metab 2016;18:1041-4.

38. Burt MG, Drake SM, Aguilar-Loza NR, et al. Efficacy of a basal bolus insulin protocol to treat prednisolone-induced hyperglycaemia in hospitalised patients. Intern Med J 2015;45:261-6.

39. Dhital SM, Shenker Y, Meredith M, et al. A retrospective study comparing neutral protamine hagedorn insulin with glargine as basal therapy in prednisone-associated diabetes mellitus in hospitalized patients. Endocr Pract 2012;18:712-9.

40. Gosmanov AR, Goorha S, Stelts S, et al. Management of hyperglycemia in diabetic patients with hematologic malignancies during dexamethasone therapy. Endocr Pract 2013;19:231-5.

41. Umpierrez GE, Hellman R, Korytkowski MT, et al. Endocrine Society. Management of hyperglycemia in hospitalized patients in non-critical care setting: an endocrine society clinical practice guideline. J Clin Endocrinol Metab 2012;97:16-38.

42. American Diabetes Associat. Standards of Medical Care in Diabetes. Diabetes Care 2016;39(Suppl 1):1-119.

43. Management of Hyperglycaemia and Steroid (Glucocorticoid) Therapy. Joint British Diabetes Societies for inpatient care 2014.

44. Carol Jairam NO. Steroid Therapy and Management of Hyperglycaemia. 2010.

45. Malcolm J, Halperin I, Miller DB, et al. In-Hospital Management of Diabetes. Can J Diabetes 2018;42 Suppl 1:S115-S123.

46. Clore JN, Thurby-Hay L. Glucocorticoid-induced hyperglycemia. Endocr Pract 2009;15:469-74.

47. Baldwin D, Apel J. Management of hyperglycemia in hospitalized patients with renal insufficiency or steroid-induced diabetes. Curr Diab Rep 2013;13:114-20.

48. Suh S, Park MK. Glucocorticoid-Induced Diabetes Mellitus: An Important but Overlooked Problem. Endocrinol Metab 2017;32:180.

49. Khazai NB, Hamdy O. Inpatient Diabetes Management in the Twenty-First Century. Endocrinol Metab Clin North Am 2016;45(4):875-94.

50. Gosmanov AR. A practical and evidence-based approach to management of inpatient diabetes in non-critically ill patients and special clinical populations. J Clin Trans/ Endocrinol 2016;5:1-6. 
51. Umpierrez GE, Smiley D, Zisman A, et al. Randomized study of basal-bolus insulin therapy in the inpatient management of patients with type 2 diabetes (RABBIT 2 trial). Diabetes Care 2007:30:2181-6.

52. Umpierrez GE, Hellman R, Korytkowski MT, et al. Management of hyperglycemia in hospitalized patients in non-critical care setting: an endocrine society clinical practice guideline. J Clin Endocrinol Metab 2012;97:16-38

53. Browning LA, Dumo P. Sliding-scale insulin: an antiquated approach to glycemic control in hospitalized patients. Am J Health Syst Pharm 2004;61:1611-4.

54. Roberts GW, Aguilar-Loza N, Esterman A, et al. Basal-bolus insulin versus sliding-scale insulin for inpatient glycaemic control: a clinical practice comparison. Med J Aust 2012;196:266-9.

55. Brady VJ, Grimes D, Armstrong T, et al. Management of steroidinduced hyperglycemia in hospitalized patients with cancer: a review. Oncol Nurs Forum 2014;41:E355-E365.
56. Lepore M, Pampanelli S, Fanelli C, et al. Pharmacokinetics and pharmacodynamics of subcutaneous injection of long-acting human insulin analog glargine, NPH insulin, and ultralente human insulin and continuous subcutaneous infusion of insulin lispro. Diabetes 2000;49:2142-8.

57. Seggelke SA, Gibbs J, Draznin B. Pilot study of using neutral protamine Hagedorn insulin to counteract the effect of methylprednisolone in hospitalized patients with diabetes. $J$ Hosp Med 2011;6:175-6.

58. Jairam C, Oliver N. Steroid Therapy and Management of Hyperglycaemia. Imperial College Clinical Guidance NHS Trust 2010 http://www.imperialendo.co.uk/Steroids.

59. Draznin B, Gilden J, Golden SH, et al. Pathways to quality inpatient management of hyperglycemia and diabetes: a call to action. Diabetes Care 2013;36:1807-14. 